\title{
Imaging of hyperalgesia in rats by functional MRI
}

\author{
Andreas Hess ${ }^{\mathrm{a}, *, 1}$, Marina Sergejeva ${ }^{\mathrm{a}, 1}$, Lubos Budinsky ${ }^{\mathrm{b}}$, \\ Hanns Ulrich Zeilhofer ${ }^{\mathrm{a}}$, Kay Brune ${ }^{\mathrm{a}, \mathrm{b}}$ \\ ${ }^{a}$ Department of Experimental and Clinical Pharmacology and Toxicology, Institute for Pharmacology and Toxicology, Fahrstrasse 17, D-91054 \\ Erlangen, Germany \\ b Innovations in Animal and Consumer Protection, Fahrstrasse 17, D-91054 Erlangen, Germany
}

Received 6 July 2005; received in revised form 12 January 2006; accepted 27 January 2006

Available online 6 March 2006

\begin{abstract}
Cerebral activation in response to sequences of temperature boosts at the hindpaw was observed in functional magnetic resonance imaging (fMRI) experiments in isoflurane anesthetized rats. Cingulate, retrosplenial, sensory-motor and insular cortex, medial and lateral posterior thalamic nuclei, pretectal area, hypothalamus and periaqueductal gray were the most consistently, often bilaterally activated regions. With the same experimental paradigm, activity changes in the brain following subcutaneous zymosan injection into one hindpaw were detected. These changes developed over time (up to $4 \mathrm{~h}$ ) in parallel with the temporal development of hyperalgesia shown by a modified Hargreaves test, thus reflecting processes of peripheral and central sensitization. When the heat stimuli were applied to the inflamed paw, the hyperalgesia manifested itself as a volume increase of the activated areas and/or an enhanced functional blood oxygenation level dependent (BOLD) signal in all the above-mentioned brain regions. Enhanced BOLD signals were also observed in response to stimulation of the contralateral non-injected paw. They were significant in higher associative regions and more pronounced in output-related than in input-related brain structures. This indicates additional sensitization processes in the brain, which we named cerebral sensitization.

Long lasting zymosan-induced hyperalgesia could be monitored with high resolution fMRI in rats under isoflurane anaesthesia. This technique may provide an effective method for testing new analgesics and studying structure specific pain processing. (C) 2006 European Federation of Chapters of the International Association for the Study of Pain. Published by Elsevier Ltd. All rights reserved.
\end{abstract}

Keywords: Functional MRI; Rat; Brain; Hyperalgesia; Sensitization; Zymosan

\section{Introduction}

The need for novel analgesics remains. Testing them has so far involved behavioural experiments on animals with acute tissue damage, such as the well established formalin, tail-flick or hot plate tests (D'Amour and Smith, 1941; Woolfe and MacDonald, 1944; Dub-

\footnotetext{
* Corresponding author. Tel.: +49 913185 22003/22801; fax: +49 91318522774.

E-mail address: hess@pharmakologie.uni-erlangen.de (A. Hess).

1 These authors contributed equally to this work.
}

uisson and Dennis, 1977). Although useful, they suffer from severe drawbacks: they are subjective, require large numbers of animals and are highly demanding to them.

During the last decade, functional magnetic resonance imaging (fMRI) has proven to be an effective pain research tool in human studies (Davis et al., 1995, 1997, 1998; Vogt et al., 1996; Binkofski et al., 1998; Jones et al., 1998; Porro et al., 1998; reviews: Hudson, 2000; Peyron et al., 2000). It is also presently being explored in animals. Rat brain activation has been demonstrated with fMRI after electrical (Hyder et al., 1994; Gyngell 
et al., 1996; Tuor et al., 2000; Chang and Shyu, 2001) and chemical (formalin injection) stimulation of the rat paw (Tuor et al., 2000, 2002). Responses to capsaicin injections or following mechanical allodynia have also been investigated by fMRI (Malisza and Docherty, 2001; Malisza et al., 2003). However, the description of activated brain structures remains incomplete due to the size and anatomical peculiarities of rodent brains. Moreover, animals have often been anesthetized using $\alpha$-chloralose which is known to interfere with nociceptive signalling (Ueki et al., 1988) and does not permit repetitive studies.

Nevertheless, rat brain regions activated by nociception generally correspond to those reported for humans in different imaging studies (for reviews see: Treede et al., 1999; Hudson, 2000; Peyron et al., 2000). The structures most frequently mentioned in human pain studies are: cingulate, somatosensory, insular, parietal and prefrontal cortex, lateral and medial thalamus, hypothalamus, hippocampus, periaqueductal gray. The human PET investigation by Lorenz et al. (2002) is of special importance to this work. The authors described a specific activation of the medial thalamic pathway to the frontal lobe during heat stimulation of capsaicin treated skin indicating a strong emotional-affective component of allodynic pain.

Zymosan is an irritant causing long lasting local inflammation and central sensitization. The hyperalgesia induced gradually develops within hours and lasts for several days (Meller and Gebhart, 1997). Therefore, it is widely employed in investigations of mechanical and thermal allodynia and hyperalgesia (Sweitzer et al., 1999; Belichard et al., 2000; Gühring et al., 2000, 2001; Depner et al., 2003; Harvey et al., 2004).

Here we report for the first time an fMRI investigation of zymosan-induced hyperalgesia and central sensitization developing with time in rats under harmless isoflurane anaesthesia. In order to disentangle contributing processes, we applied a mild noxious heat stimulus to inflamed as well as non-inflamed hindpaws. Modern scan protocols allowed for a more precise identification and quantification of pain relevant structures in the rodent brain (compare Tuor et al., 2002; Malisza and Docherty, 2001). Additionally, repetitive application of phasic stimuli in our experiments allowed for increased statistical reliability.

\section{Materials and methods}

Wistar male rats weighing ca. $350 \mathrm{~g}$ were bred at the Institute for Experimental and Clinical Pharmacology and Toxicology. In all experiments, the ethical guidelines for investigation of experimental pain in conscious animals were followed (Zimmermann, 1983). The experiments were approved by the local ethics committee.

\subsection{Behavioural experiment}

A modified Hargreaves test (Hargreaves et al., 1988) was applied to assess thermal hyperalgesia in the rats. The observation cage with individual $21 \times 17 \times 14 \mathrm{~cm}$ boxes and a metal grid bottom instead of a glass floor was used. Paw withdrawal latencies (PWL) were determined on exposure of the hindpaws to a defined thermal stimulus using a commercially available apparatus (Hargreaves Test UGO BASILE Biological Research Apparatus, Comerio, Italy).

Rats $(n=4)$ were placed in the test boxes $2 \mathrm{~h}$ prior to the experiment to allow for adaptation. Then the baseline PWLs were measured for the both hindpaws. After that, unilateral hindpaw inflammation was induced by subcutaneous injection of $0.15 \mathrm{mg}$ zymosan A (Sigma, Deisenhofen, Germany) in $50 \mu \mathrm{lBS}$ into the right hindpaw. PWLs of the both hindpaws were determined 1, 2, 3,4 and $5 \mathrm{~h}$ after the injection. After the experiment the animals were sacrificed with $\mathrm{CO}_{2}$ to prevent further pain. Thermal hyperalgesia at every time point was assessed as an averaged percentage difference between the PWSs of the right (injected) and left (non-injected) paws according to the formula $((R \times L) / L) \times 100 \%$. Corresponding significances of the PWL latency decrease were evaluated by a Student's $t$-test.

\subsection{FMRI experiment}

\subsubsection{Animal preparation}

Each animal was initially anesthetized with isoflurane $(5 \%)$ in medical air $(400-450 \mathrm{ml} / \mathrm{min})$ and was placed on a specially designed Plexiglas tray. The animal body temperature was kept constant at $37^{\circ} \mathrm{C}$ by warm water circulating in the tray. The head of the animal was firmly fixed with a special "nose-mouth mask" directly below the surface coil, and isoflurane anaesthesia continued through this mask. The fixation stability was controlled by a rapid MR measurement (see below) thus providing no major motion artefacts during the whole measurements.

The vital functions were monitored during the whole experiment via a breathing sensor fixed under the chest of the rat and by measuring the body temperature. Slight changes of the isoflurane supply (between $1 \%$ and $2 \%$ made it possible to keep the respiration rate constant at $60 / \mathrm{min}$ in the periods without heat stimulation leading to a constant blood $\mathrm{pCO}_{2}$ level of (38 $\mathrm{mmHg} \pm 10 \%$, compare Ramos-Cabrer et al., 2005) during the whole experiment. The $\mathrm{pCO}_{2}$ level was measured transcutaneously by a pediatric blood gas monitor (TCM3, Radiometer Copenhagen).

In the control experiments $(n=4), 50 \mu$ PBS were subcutaneously injected into the right hindpaw immediately before the animal was placed on the tray. In zymosan hyperalgesia experiments $(n=8)$, the same amount 
of zymosan A as in the behavioural experiment $(0.15 \mathrm{mg}$ in $50 \mu \mathrm{PBS}$ ) was injected in the same way. The subsequent preparation for the fMRI measurement took $30-40$ min, a time in which also a constant $\mathrm{pCO}_{2}$ level was reached (cf. Ramos-Cabrer et al., 2005).

After MRI experiments, the animals which had a zymosan injection were sacrificed with an overdosage of isoflurane; those animals which did not receive zymosan were returned to their cages.

\subsubsection{Heat stimulation}

The contact heat stimulation was performed using the custom made computer controlled Peltier heating and cooling device (MRI-ThS1-2ch). The stimulation system does not introduce any disturbances in the MR scanning and is also not influenced by the gradient pulses. Two Peltier elements with $15 \times 15 \mathrm{~mm}$ surface were fixed at the both hindpaws. Starting at a baseline of $34{ }^{\circ} \mathrm{C}$ the stimulation temperature of $46^{\circ} \mathrm{C}$ was reached after $15 \mathrm{~s}$ with $0.8^{\circ} \mathrm{C} / \mathrm{s}$. The stimulation temperature plateau was held for $5 \mathrm{~s}$. Within the following $15 \mathrm{~s}$ the temperature dropped linearly back to the baseline. Stimuli were applied at the left and the right paw in an alternating sequence at intervals of $3 \mathrm{~min} 25 \mathrm{~s}$. In no case was burning of the paw observed either directly after the experiment or during the next 2 days of observation for the control group.

\subsubsection{MR measurements}

FMRI experiments were performed on a $4.7 \mathrm{~T}$ BRUKER Biospec scanner with a free bore of $40 \mathrm{~cm}$, equipped with an actively RF-decoupled coil system. A whole-body birdcage resonator enabled homogenous excitation, and a $3 \mathrm{~cm}$ surface coil, located directly above the head of the animal to maximize the signalto-noise-ratio, was used as a receiver coil. The scanning procedure started with the acquisition of $\mathrm{T} 2$ weighted spin echo horizontal anatomical reference images (slice thickness $1 \mathrm{~mm}$, field of view $35 \times 35 \mathrm{~mm}$, matrix $256 \times 128$, TR $=2800 \mathrm{~ms}$, TEef $=77 \mathrm{~ms}$ ) using a rapid acquisition relaxation enhanced sequence (RARE, Henning et al., 1986). Functional images were acquired using Echo Planar Technique (EPI). First, a set of 300 axial single slice scans with a high time resolution (200 ms per scan) was acquired (slice thickness $1 \mathrm{~mm}$, field of view $25 \times 25 \mathrm{~mm}$, matrix $64 \times 64$, TR $=200 \mathrm{~ms}$, TEef $=23.4 \mathrm{~ms}$ ). The resulting movie (five frames per second) was evaluated visually. In case of visible motion artifacts, the animal was remounted. Afterwards a functional series of 1800 sets of seven axial EPI images (slice thickness $1 \mathrm{~mm}$, field of view $25 \times 25 \mathrm{~mm}$, matrix $64 \times 64, \mathrm{TR}=2000 \mathrm{~ms}$, TEef $=23.4 \mathrm{~ms}$ ) was acquired. Slice 2 was positioned at Bregma $-4.16 \mathrm{~mm}$ according to Paxinos and Watson' rat brain atlas (1998) using as the anterior-posterior reference the smallest distance between the posterior tip of the corpus striatum and the anterior tip of the hippocampus on the horizontal anatomical references images. Altogether the slices covered a central brain area from Bregma 1.20 to $-5.20 \mathrm{~mm}$ according to Paxinos.

The initial 120 scans covered a 4-min period without any stimulation. Then there followed 14 cycles of stimulation alternating between right and left hindpaw. Each cycle consisted of 120 scans (covering $35 \mathrm{~s}$ of heating and $3 \mathrm{~min} 25 \mathrm{~s}$ no stimulation). Altogether one such session lasted $1 \mathrm{~h}$. Finally, a set of anatomical scans with a high spatial resolution in the same area as the functional set was acquired with RARE (slice thickness $1 \mathrm{~mm}$, field of view $25 \times 25 \mathrm{~mm}$, matrix $256 \times 256$, TR $=400 \mathrm{~ms}$, $\mathrm{TE}=18 \mathrm{~ms}, \mathrm{NEX}=8$ ).

We performed two imaging sessions on each animal in the control as well as in the zymosan group as follows: the first one starting 30-40 min after the injection, so that these experiments were all finished within $2 \mathrm{~h}$ or less (they will be referred to here as control-2h and zymo- $2 \mathrm{~h}$ sessions), and the second one $-60 \mathrm{~min}$ after the first session - so that these measurements were completed within $4 \mathrm{~h}$ after the injection (control-4h and zymo-4h sessions). According to our behavioural findings, the zymosan-induced hyperalgesia was assumed to be fully developed within $4 \mathrm{~h}$ (compare Meller and Gebhart, 1997).

\subsubsection{Data processing}

Functional analysis was performed using BrainVoyager 2000 (V 4.8.5.0). In some cases the motion correction algorithm implemented in BrainVoyager was applied. The motion detected was always clearly below 1 pixel due to the stable fixation of the rat head. The first five data volumes were discarded from further analysis in order to exclude saturation effects.

Cross-correlation analysis of the changing amplitude of the BOLD signal was performed with the stimulation boxcar function (no time-lag), where the first 60 scans ( $2 \mathrm{~min}$ ) of each stimulation cycle where taken as stimulation phase - a little longer than the physical stimulation, to cover the whole BOLD response. The resulting $r$ values of the Pearson's correlation coefficients were transformed to $t$ values by Fisher transformation and subsequently subjected to a Student's $t$-test (Bandettini et al., 1993) with the null hypothesis of no significant correlation with the stimulation paradigm. The threshold for the $p$-values was chosen to be $\leqslant 0.04$ (Hess et al., 2000). Different groups of activated voxels were labelled as belonging to certain brain structures. Since the slice position of our individual scans were already registered (see MRI measurements) we were able to identify brain structures by overlaying the corresponding images from the Paxinos rat brain atlas (Paxinos and Watson, 1998). Single subject quantification of these activated voxel groups regarding volume, amplitude (BOLD percentage signal changes) and time 
profiles of the activated structures was obtained by the custom analysis program MRIan written in IDL. The following pre-processing steps were applied. Firstly, a baseline correction was performed by subtracting the mean of the first 30 acquisitions from all the acquisitions. Secondly, a least-square polynomial $\left(8^{\circ}\right)$ trend line correction was carried out in order to remove low frequency changes over the long recording time of $1 \mathrm{~h}$. Note that no further smoothing in space or time at this stage was applied. The volumes of the activated regions were determined as the number of voxels for each specific label (structure).

In order to be able to follow hyperalgesia in terms of increased BOLD signal amplitudes over time in topographically identical regions, the labels from the datasets of control-4h and zymo-4h were applied to the control$2 \mathrm{~h}$ and zymo- $2 \mathrm{~h}$ datasets, respectively.

Averaged time profiles of the heat stimulus evoked BOLD signals were generated for each structure in each animal for the both sessions, i.e., at 2 and $4 \mathrm{~h}$. The BOLD percentage signal change of the activated voxels of each stimulus presentation was averaged over the time of 10 acquisitions before, 60 during and 10 acquisitions after each stimulation cycle. This was done seperately for the stimulation of the injected and non-injected paw. For better description of the peak the averaged time profiles were smoothed by a central running mean filtering (filter width $=5$ ). Consequently, the amplitude of the percentage BOLD signal change was determined as the peak of the averaged time profile.

The rationale of our statistical testing was the following. First, in order to asses global time-dependent effects on activated volumes and peak signal changes we performed an analysis of variance (ANOVA) with time and region as within-subject factors and treatment (control/zymosan) as between-subject factor. Next, in order to check for significant differences in volume and peak due to the injection per se and/or due to the long experimental time more specifically, we tested control- $2 \mathrm{~h}$ versus control-4h group. To estimate if zymosan induces changes already within $2 \mathrm{~h}$, we tested control-2h versus zymo-2h. To follow further development of zymosaninduced effects we tested zymo-2h versus zymo- $4 \mathrm{~h}$. These three evaluations were carried out for both activated volume and peak value with a two-tailed Student's $t$-test: the first and the third one with a paired $t$-test, the second one with an unpaired $t$-test.

\section{Results}

\subsection{Behaviour}

Administration of zymosan evoked thermal hyperalgesia developing over time. Hyperalgesia was significant
2, 3, 4 and $5 \mathrm{~h}$ after the injection. PWL decreases reached its maximum in about $3 \mathrm{~h}$ (Fig. 1).

\subsection{FMRI}

In spite of the well known high individual variation of activated structures, especially in pain fMRI (Hudson, 2000; Peyron et al., 2000), we found reproducible and quantifiable signals allowing for functional imaging of nociception related brain activation in rats.

\subsubsection{Nociception activated brain structures}

In control experiments when heat was applied to uninflamed skin we saw a stimulus evoked, often bilateral activation of many cortical and subcortical structures, such as: cingulate, retrosplenial, sensory-motor, insular, piriform, rhinal and parietal association cortices, pretectal area, basal ganglia, hippocampus, septal area, thalamus, hypothalamus, periaqueductal gray. Within the thalamus we found activation in the areas of the following nuclei or nuclear groups: habenular complex, anterior group, the group of nuclei, which functionally belong to the medial system of thalamus (Treede et al., 1999; Hudson, 2000), ventral posterior lateral nucleus belonging to the lateral system of thalamus, paraventricular nucleus, lateral posterior nucleus, lateral geniculate nucleus. We focused on the areas which were most consistently activated. The majority of them could be defined as right ( $r)$ - ipsilateral or left (1) - contralateral to the injection side, some of them, located at the border between two hemispheres, could not. The areas of our interest were: cingulate cortex (C), retrosplenial cortex (RS), primary somatosensory cortex (S1-r, S1-1), secondary somatosensory cortex (S2-r, S2-1), motor cortex (M-r, M-1), insular cortex (I-

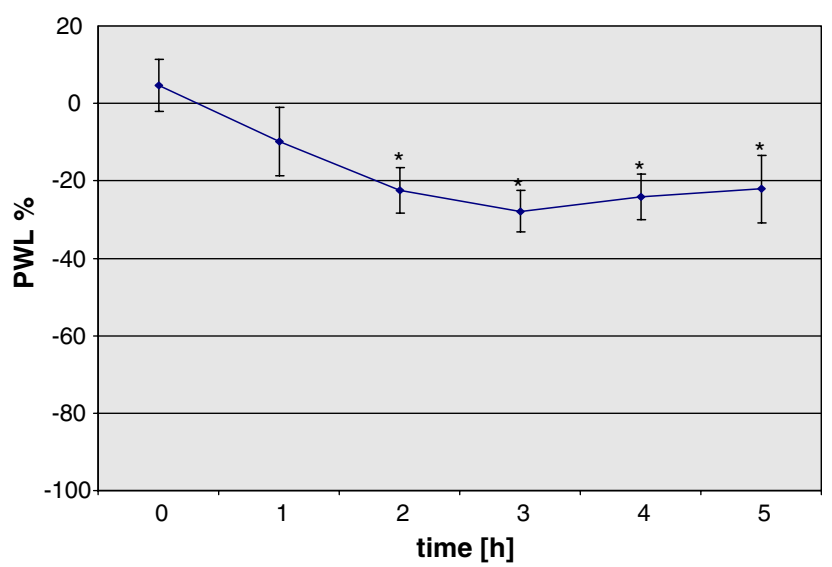

Fig. 1. Time course of the paw withdrawal latency difference in response to thermal stimulation of the right (injected) and left (noninjected) hindpaws. Data are presented as a relative difference between the zymosan injected right and the non-injected left hindpaw calculated as: $((R \times L) / L) \times 100$. Significant differences were calculated by Student's $t$-test. 
r, I-1), pretectal area/lateral posterior thalamic nucleus (PTA/LP-r, PTA/LP-1), medial thalamus (MT), hypothalamus (HT-r, HT-1), periaqueductal gray (PAG), as exemplified in Fig. 2. Areas S2-r, M-1 and I-r were later excluded from the statistic evaluation because of their insufficient representation in the control group. Some structures were difficult or impossible to separate due to their close proximity and the limited spatial resolution. For instance, pretectal nuclei could not be distinguished from the neighbouring lateral posterior thalamic nucleus, and the thalamic medial system - from habenular and anterior nuclear groups. So, the term "MT" includes not only the medial thalamic system, but also habenular and anterior nuclear groups partly or as a whole.

\subsubsection{Changes of the activated volumes}

In saline-injected animals, comparison of the volumes, activated by the heat stimulus in control-2h and control-4h groups revealed a trend towards a decrease in many brain areas (Fig. 3). This decrease was even highly significant in the following areas: MT, S1-r.
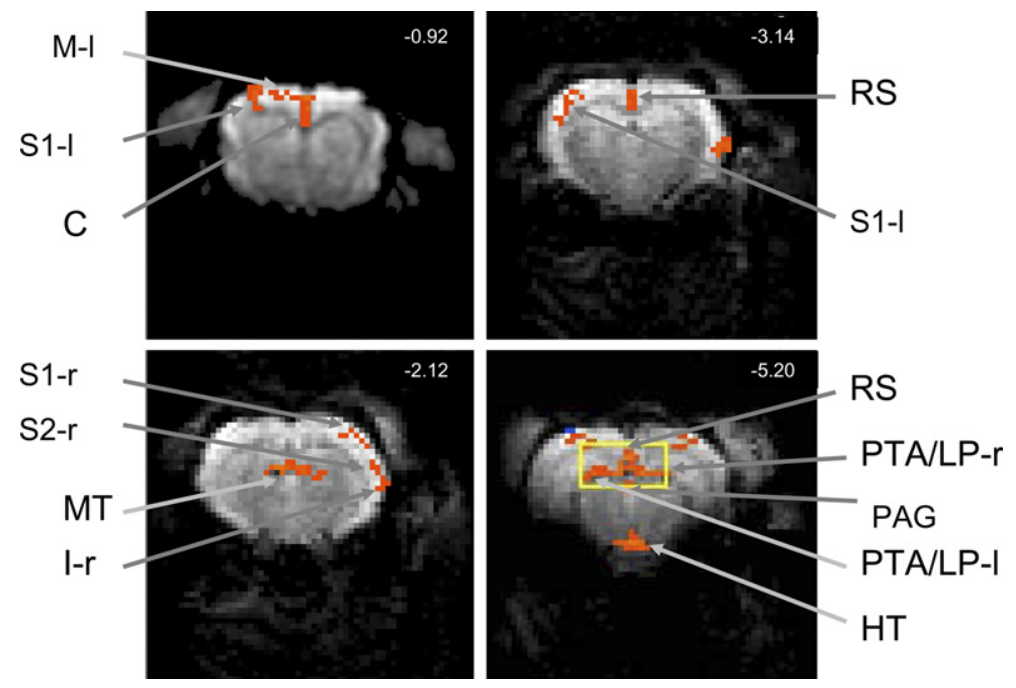

Fig. 2. Brain structures activated by mild noxious heat stimulation of the hindpaws, examples from different animals. Numbers (right upper corners) - coordinates with respect to bregma (mm), adopted from Paxinos and Watson (1998). Medial thalamic system: MT; pretectal area/lateral posterior nucleus: PTA/LP; cingulate cortex: C; retrosplenial cortex: RS; primary somatosensory cortex: S1; secondary somatosensory cortex: S2; motor cortex: M; insular cortex: I; hypothalamus: HT; periaqueductal gray: PAG; left: 1; right: r.

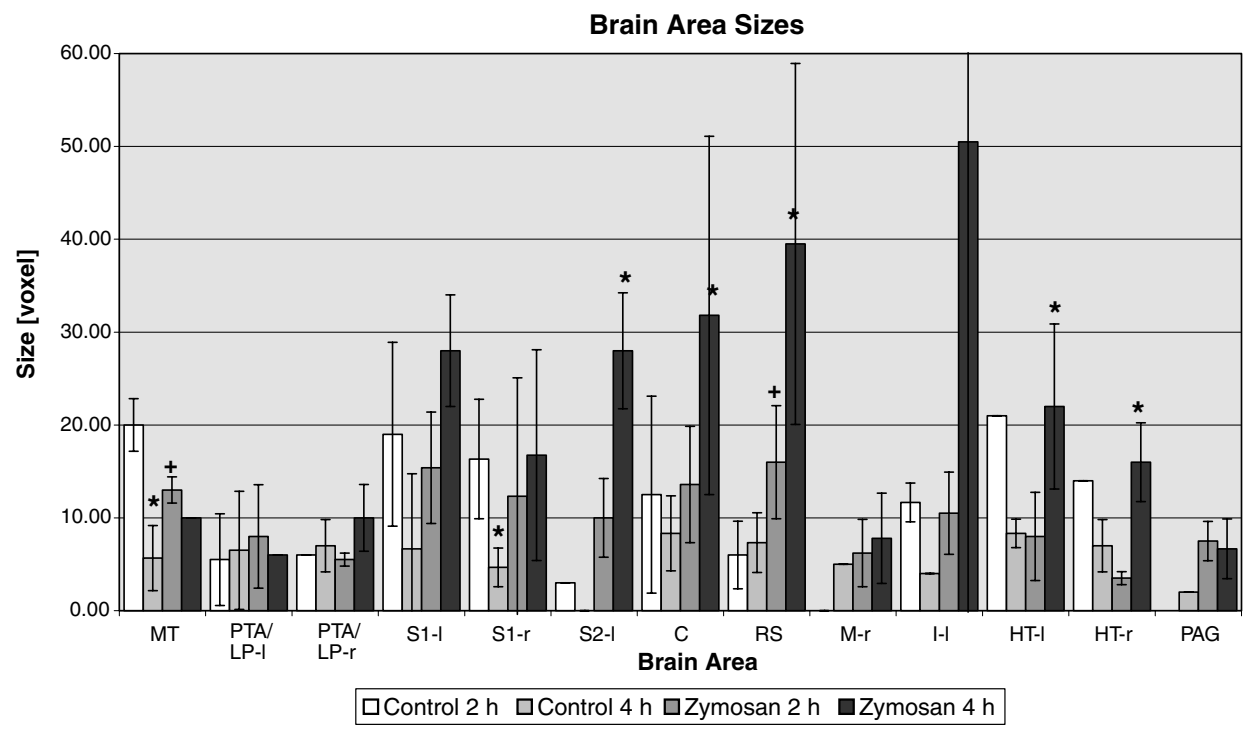

Fig. 3. Volumes of the activated areas in the control-2h (white), control-4h (grey), zymo-2h (dark grey) and zymo-4h (black columns) sessions. Abbreviations as in Fig. 2. Significant differences (Student's $t$-test) are given by plus for the comparison of the control versus zymo group and asterisks for significant differences within each group. 

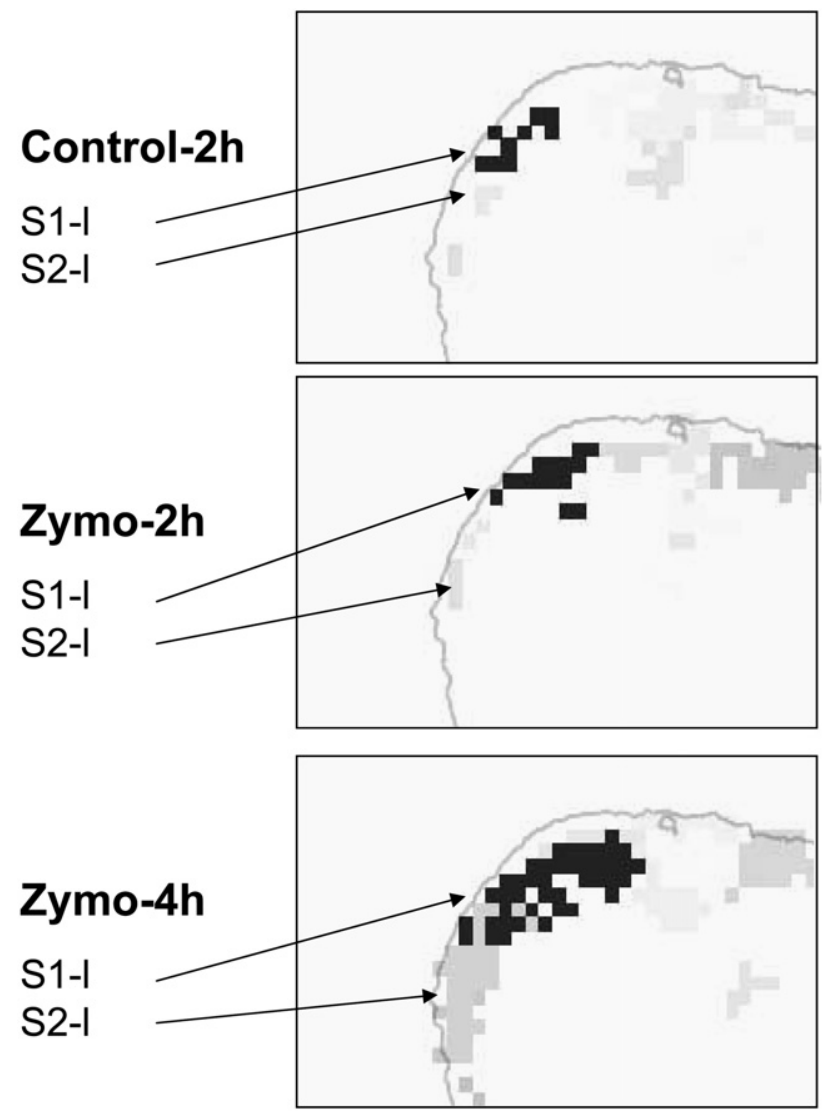

Fig. 4. Comparison of the areas activated by mild noxious heating of the right (injected) hindpaw in the contralateral somatosensory cortex between control-2h, zymo- $2 \mathrm{~h}$ and zymo- $4 \mathrm{~h}$ sessions, examples. Note the large increased area of the primary (S1-1, dark grey) as well as secondary (S2-1, light grey) somatosensory cortex during developing hyperalgesia. Areas are generated by projections along the anteriorposterior axis through the slices. Increase in activated area of other structures, e.g., C, cannot be seen due to their extension along the anterior-posterior axis, but quantification of their activated volume is given in Fig. 3.
The ANOVA revealed a global zymosan dependent change in activated volume $F(3,100)=18.4, p<0.0001$ for time and region $\times$ treatment.

The $t$-tests performed showed, that there were no clear significant differences in activated volumes between the control-2h and the zymo- $2 \mathrm{~h}$ groups indicating that hyperalgesia did not show up in the sizes of the activated volumes within $2 \mathrm{~h}$.

In contrast, there was an increase in the activated volumes in the zymo- $4 \mathrm{~h}$ session, compared to the zymo- $2 \mathrm{~h}$ session, in most of the investigated areas (Fig. 3). The most dramatic volume increases (2-5 times) were observed in the contralateral cortical structures S1-1, S2-1 (cf. Fig. 4), I-1, in associative areas C, RS and bilaterally in hypothalamus (HT-1 and HT-r), most of them significant (cf. Fig. 3). In the small input structure PTA/LP-r the volume increase was smaller. MT, PTA/ LP-1 and PAG showed an insignificant volume decrease.

\subsubsection{Changes of the BOLD signal amplitudes}

The time pattern of the BOLD signals from the activated areas correlated well with the stimulation boxcar function. As an example we give the time profile of activation of the cingulate cortex $(\mathrm{C})$ averaged across all the zymo-4h animals (Fig. 5). One can see an increase in the amplitude even within the zymo-4h session. In both control sessions the amplitudes were generally higher during heating the non-injected compared to the injected paw, although not significantly (Data not shown). On the contrary, in zymosan experiments BOLD signal amplitudes were mostly higher during heating of the injected paw, than of the intact one, as can be seen in Fig. 5 for $\mathrm{C}$ in every stimulation cycle.

Again, ANOVA revealed a global zymosan dependent change in the signal amplitude $F(3,100)=15.6$, $p<0.0001$ for time and region $\times$ treatment.

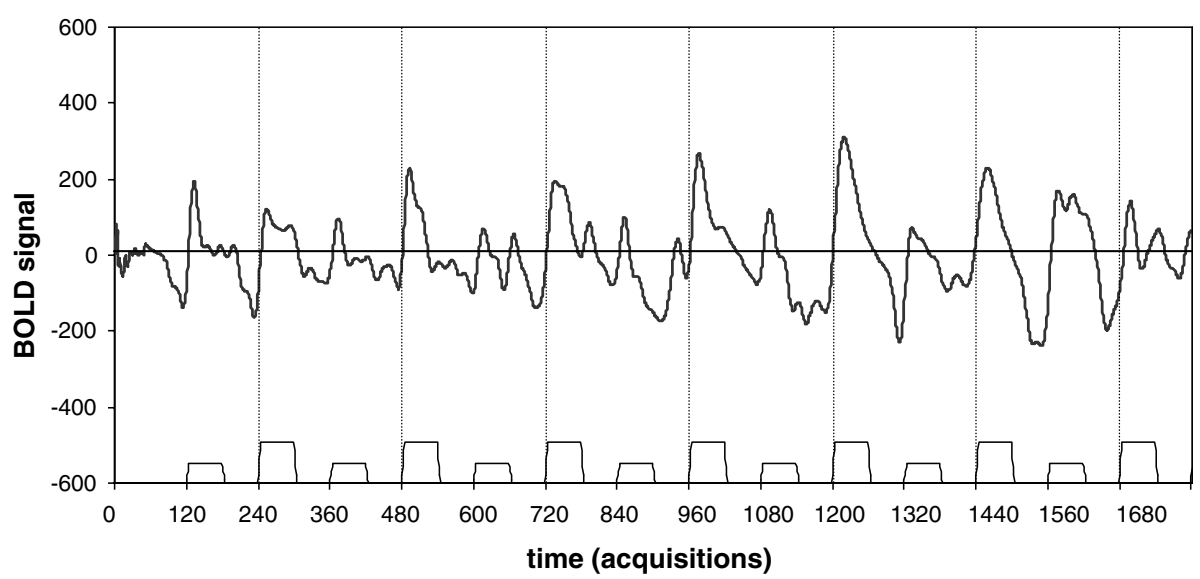

Fig. 5. Time plot of the BOLD signal after baseline and trend correction in cingulate cortex in the zymo-4h session, averaged across eight animals. Rectangular pattern shows the time periods of the heat stimulation, the lower plateaus indicate stimulation of the intact paw, the higher ones stimulation of the zymosan injected paw. Enhanced BOLD signal can be seen in both cases, but especially by stimulation of the injected paw. BOLD signal continues to increase within the zymo-4h session. Average data plotted are smoothed in this case by a Savitzky-Golay filter (kernel width: 32 ; order: 0 and degree 8 ). 
However, in the control experiments there were almost no changes of the BOLD signal amplitudes with time, neither for stimulation of the injected, nor for the non-injected paw as shown by the $t$-test.

Zymosan initiated major changes of the heat stimulus related activity, developing over time. Heating the injected paw increased the BOLD signal amplitude noticeably in all the brain areas investigated already $2 \mathrm{~h}$ after injection (Fig. 6, comparison between control-2h and zymo-2h). The difference reached the level of significance in PTA/LP-r, S1-1, S1-r, C, RS, M-r, I-1, HT-1, HT-r, PAG.

No consistent trend in the BOLD signal changes was observed in the control- $2 \mathrm{~h}$ versus zymo- $2 \mathrm{~h}$ comparison following heating of the non-injected paw (cf. Fig. 7). Significant differences were only in M-r, I-l and PAG.

Zymo-2h/zymo-4h comparison for the stimulation of the injected paw showed a further increase of the BOLD signal amplitude in all the areas (Fig. 6), significant in PTA/LP-r, S1-1, S1-r, C, RS, M-r, HT-1 and HT-r.

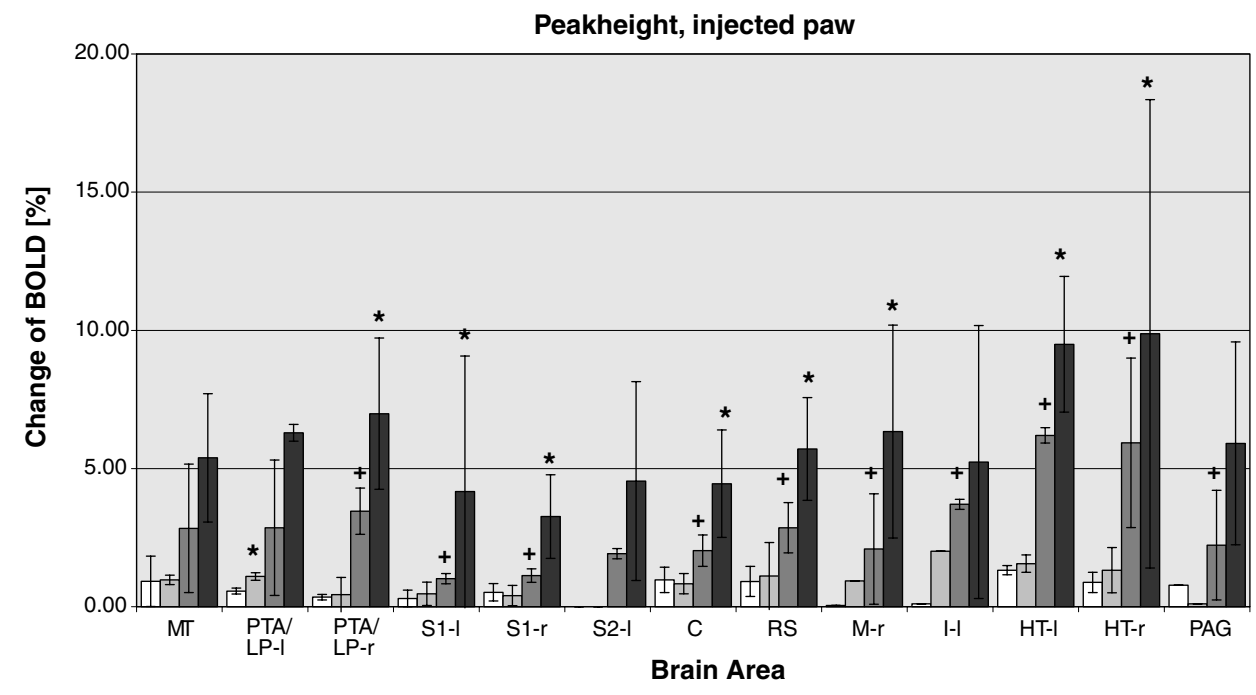

$\square$ Control $2 \mathrm{~h}$ पControl $4 \mathrm{~h}$ 口Zymosan $2 \mathrm{~h}$ 口Zymosan $4 \mathrm{~h}$

Fig. 6. Relative amplitudes of the BOLD signal following the stimulation of paws injected with saline (control-2h: white; control-4h: grey) and zymosan (zymo-2h: dark grey; zymo-4h: black columns). Plus indicate significance of the differences (Student's $t$-test) between control and zymo group and asterisks for the within group comparisons, respectively. Abbreviations like in Fig. 3.

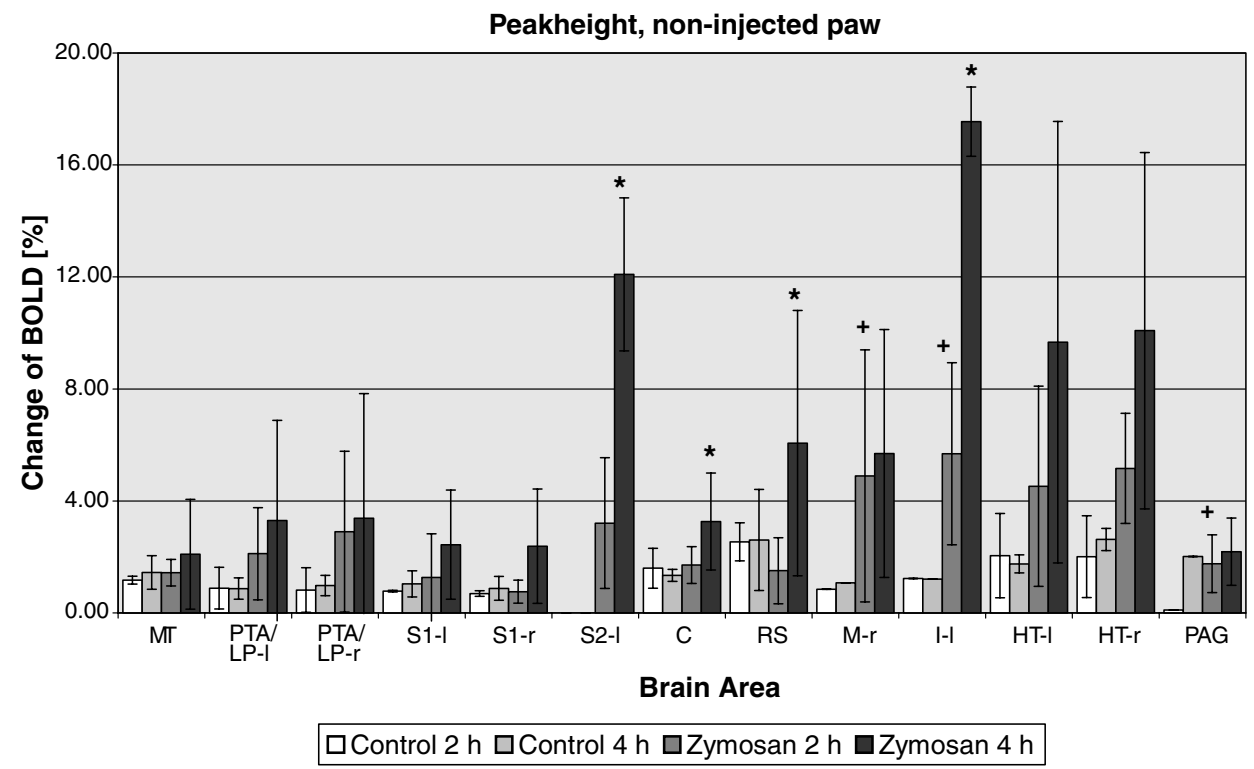

Fig. 7. Similar to Fig. 6, but for the stimulation of the non-injected paw. Note, that in this case left is ipsi- and right is contralateral to the side of stimulation. 
Moreover, by stimulation of the non-injected paw an increase of the BOLD signal amplitude from zymo-2h to zymo-4h could also be detected (Fig. 7). The increase reached significance in higher order brain areas like the S2-1, C, RS and I-1 and was also high in HT-1, and HT-r, indicating processes of central sensitization.

Both increases in volume and amplitude coincided in time with the development of zymosan-induced hyperalgesia (own behavioural data, Meller and Gebhart, 1997; Sweitzer et al., 1999; Belichard et al., 2000; Gühring et al., 2000, 2001; Depner et al., 2003; Harvey et al., 2004).

\section{Discussion}

Zymosan-induced rat paw inflammation is a widely used model of chronic pain and hyperalgesia. It develops within hours and lasts for up to a fortnight. Our behavioural experiment demonstrated, that even a very small dose of zymosan used $(0.15 \mathrm{mg}$; compare: Meller and Gebhart, 1997) evokes thermal hyperalgesia lasting at least $4-5 \mathrm{~h}$. Applying this small dose we were able to detect structure specific brain responses going along with early hyperalgesia by non-invasive imaging (BOLD fMRI). Applying heat stimuli to inflamed but also to non-inflamed paws, we were able to discern different processes of central sensitization. Several aspects of this investigation deserve discussion in detail.

\subsection{Technical aspects}

We report a higher degree of spatial and temporal resolution than in previous rat fMRI studies (e.g., Tuor et al., 2000; Malisza et al., 2003; Chang and Shyu, 2001). These studies used slow imaging sequences (RARE, FLASH), limiting the time resolution to minutes. Moreover, only 2-4 slices could be scanned. In human fMRI EPI recording is now standard, providing high spatial and temporal resolution (hundreds of milliseconds). For EPI in animal imaging special requirements have to be met due to higher field strength and smaller sample size. By introducing EPI to animal pain fMRI in this study we reached a higher completeness of identified structures throughout the brain. Dedicated surface coil permitted detection of the BOLD signal not only from cortical areas, like in other rat fMRI studies (Hyder et al., 1994; Gyngell et al., 1996; Scanley et al., 1997; Tuor et al., 2000; Malisza et al., 2003), but also from deep cerebral structures. Furthermore, the high temporal resolution of EPI enabled us to present short stimuli repetitively thereby increasing statistical power. However, there is still a need for further technical improvement. BOLD fMRI does not reach the precision of autoradiographic mapping methods which are still the gold standard with respect to spatial resolution. On the other hand, the autoradiographic methods used in animal pain studies have time resolutions of $5 \mathrm{~min}$ (Paulson et al., 2002) up to $45 \mathrm{~min}$ (Mao et al., 1993; Neto et al., 1999; Porro et al., 1999) and do not allow detection of short repetitive stimulus induced responses. Therefore, these studies show long lasting generalized pain responses following only one particular stimulation. Mao et al. (1993) speculate about the bilateral activation being at least partly a result of ongoing normal physiological and regulatory activity found in a unilateral pain model.

Effects of anaesthesia on brain functions are still a critical issue, especially for investigations of supraspinal nociception. Alpha-chloralose, most frequently applied in rat fMRI, overproportionally increases glucose metabolism and local cerebral blood flow in cortical regions following electrical stimulation of somatic sites (Ueki et al., 1988) thus blurring the BOLD signal. It allows only for slow recovery and impairs animal health (pneumonia) prohibiting long term experiments. Isoflurane has been used in several animal fMRI studies (Porszasz et al., 1997; Abo et al., 2004). It allows for stable anaesthesia, fast recovery without measurable damage and repetitive experiments. It is, however, known to decrease the activity of the human somatosensory cortex (Antognini et al., 1997). We did detect nociception related activity in somatosensory cortices. We can not exclude that this activity was influenced by isoflurane; still we consider it better suited than $\alpha$-chloralose for long lasting fMRI. None of our control animals died during the experiments or during following days.

\subsection{Essential findings}

With mild noxious contact heat stimulation and fMRI we detected cerebral activation in anesthetized rats, well correlated to the boxcar stimulation pattern (Fig. 5). As expected, distribution of the activated areas was generally consistent with those known from different pain studies (review Hudson, 2000) but only partly described in $\alpha$-chloralose anesthetized rats (Tuor et al., 2000; Chang and Shyu, 2001; Malisza et al., 2003). Additionally, our paradigm allowed us to discern activation in primary sensory areas from activations in areas known to be involved in secondary sensory as well as cognitive/emotional processing (Hudson, 2000).

All investigated structures along the pain pathway showed increased activity under zymosan-induced hyperalgesia. An increase of the activated volume or of the BOLD signal amplitude, or both, with time could be observed.

\subsubsection{Volume effects}

Two hours after zymosan injection hyperalgesia was not noticeable in terms of activated volumes. There were no clear differences between control-2h and zymo- $2 \mathrm{~h}$. 
Unexpectedly, in animals injected with vehicle only, the activated volumes of many structures significantly decreased from control-2h to control-4h (Fig. 3). This could be an effect of anaesthesia, but also peripheral and/or central adaptation. Against this background the increase of activated volumes in many structures in the zymo-4h group is even more impressive (Fig. 3). The somatosensory cortex showed an expanded activation, especially on the contralateral side (Figs. 3 and 4). This could be ascribed to the sensory-discriminative component of pain perception (Treede et al., 1999; Bushnell et al., 1999). Activation of lateral thalamic nuclei (VP), directly projecting to S1 and S2, could not be seen reliably. A possible reason for this might be the complex topography of the somatosensory thalamus and the small number of excited neurons (cf. Casey, 1999). Also the well-known partial volume effect may explain lack of detectable volume increases in small structures in general (e.g., PAG).

\subsubsection{Amplitude effects}

BOLD signal amplitudes showed a considerable increase following heating of the injected paw already $2 \mathrm{~h}$ after zymosan injection. At $4 \mathrm{~h}$ the increase was even larger (Fig. 6) reflecting the development of zymosaninduced hyperalgesia and central sensitization. This parallelism to physiologic description suggests that our fMRI approach is capable of visualizing hyperalgesia and its development in anesthetized animals.

In agreement with Chang and Shyu (2001), we could show reliably enhanced activation in MT and $\mathrm{C}$ both belonging to the medial pain processing system (Treede et al., 1999), related to the affective-motivational component of pain. Medial thalamic nuclei have direct projections to anterior cingulate cortex (AC). Recently this has been demonstrated also with fMRI as an increased BOLD signal in AC after electrical stimulation of the ipsilateral MT (Shyu et al., 2004). AC is involved in the integration of affect, cognition and response selection (review: Devinsky et al., 1995) as well as modulatory control of pain perception (Petrovic and Ingvar, 2002). Activation of structures in pain experiments may reflect nociception, antinociception or both. This refers to the activation we detected in C, PAG and habenular complex (Cohen and Melzack, 1986).

\subsubsection{Affective-motivational and sensory-discriminative implications}

Recent PET human study of Lorenz et al. (2002) demonstrated a specific activation of the medial thalamic-cingular-frontal lobe pathway during capsaicininduced thermal allodynia. Despite major differences in our experimental paradigms and objects - conscious humans/allodynia and anaesthetized rats/hyperalgesia, - our results are quite in line with those of Lorenz and colleagues. This may indicate common peripheral/cen- tral mechanisms, mediating responses to traumatic pain, including stronger emotional-affective components (unpleasantness). Additionally, as distinct from Lorenz and co-authors, but in agreement with human fMRI allodynia study by Maihöfner et al. (2004), we found increased activation in the sensory-discriminative system (S1). This can be due to differences between fMRI and PET (cf. Casey, 1999), or, more likely, to the fact that Lorenz and colleagues compared normal vs equally intense allodynic pain: stimulation temperature in allodynia experiments was appropriately reduced, whereas we (also Maihöfner and co-authors) compared normal vs hyperalgesic (allodynic) pain at the same stimulus intensity, consequently leading to a stronger sensory-discriminative response in our hyperalgesia (allodynia) paradigms.

Another structure showing remarkable activity increase in our experiments was the insula. Insula, mediating between the two systems, is reliably activated in human pain imaging (Treede et al., 1999; Maihöfner et al., 2004), but was not described yet in rat fMRI studies.

A damaging stimulus often triggers autonomic and neuroendocrine responses. HT, PAG and amygdala constitute the main neural substrates for integrating aversive states and mediating these reactions (Tomaz and Graeff, 1993). In the PET study by Hsieh et al. (1995) traumatic pain compared to non-traumatic, prominently activated HT and PAG. Accordingly, we detected marked activity increases in these structures during hyperalgesia development, but not in controls (HT: activated volume: Fig. 3; HT, PAG: BOLD signal amplitude: Fig. 6).

\subsubsection{Cerebral sensitisation}

Stimulation of the non-injected paw resulted in activity increases in some pain-related structures $2-4 \mathrm{~h}$ after zymosan injection (Fig. 7). Interestingly, there were no significant changes in the brain regions involved in direct processing of nociceptive inputs (MT, S1). Brain areas, however, involved in higher order or cognitive-associative pain processing (S2, C, RS and I, cf. Hudson, 2000) as well as output-related structures (HT-1, HT-r) showed clear activity increases.

It is well known that unilateral disorders can produce bilateral effects, smaller on the contralateral side, for instance mirror pain (Melzack, 1990). Koltzenburg et al. (1999) in their review concluded that they are most likely mediated by spinal or brainstem commisural interneurons. Mirror hyperalgesia and allodynia have been reported in cases of unilateral inflammation (Spataro et al., 2004; Twining et al., 2004). We can not exclude that hyperalgesia in our experiments "irradiated" to the contralateral side across the spinal-cord/brainstem. However, the fact that the elevation of activity by stimulation of the intact paw was significant only in 
associative and considerable in output-, but not in input-related cerebral brain structures, argues against this assumption. Therefore, this response increase in associative cortical and consequently in output-related structures must have been generated in the brain, i.e., reflects cerebral sensitization.

In summary, we demonstrated for the first time, that fMRI is capable of visualizing zymosan-induced hyperalgesia at different stages of inflammation. We propose to use this model for further long lasting fMRI investigations of CNS plasticity and testing of new analgesics. Isoflurane anaesthesia appears advantageous because it allows for repetitive testing. Introducing EPI to animal pain fMRI enabled us to reach a higher completeness of identified structures of the rat nociceptive pathway and to describe an additional type of central sensitisation - cerebral sensitisation.

\section{Acknowledgements}

This study was supported by the SET project "Magnetresonanztomographie in der Schmerzforschung", BMBF project "Kopfschmerz: A2", IZKF in Erlangen and by the Doerenkamp Professorship in Innovations in Animal and Consumer Protection. The authors thank Dr. S. Kreitz for generous help in statistical evaluation and Mss. A. Püster and S. John for the excellent technical assistance.

\section{References}

Abo M, Suzuki M, Senoo A, Miyano S, Yamauchi H, Yonemoto K, et al. Influence of isoflurane concentration and hypoxia on functional magnetic resonance imaging for the detection of bicuculline-induced neuronal activation. Neurosignals 2004;13:144-9.

Antognini JF, Buonocore MH, Disbrow EA, Carstens E. Isoflurane anesthesia blunts cerebral responses to noxious and innocuous stimuli: a fMRI study. Life Sci 1997;61:PL349-54.

Bandettini PA, Jesmanowicz A, Wong EC, Hyde JS. Processing strategies for time-course data sets in functional MRI of the human brain. Magn Reson Med 1993;30:161-73.

Belichard P, Landry M, Faye P, Bachvarov DR, Bouthillier J, Pruneau $\mathrm{D}$, et al. Inflammatory hyperalgesia induced by zymosan in the plantar tissue of the rat: effect of kinin receptor antagonists. Immunopharmacology 2000;46:139-47.

Binkofski F, Schnitzler A, Enck P, Frieling T, Posse S, Seitz RJ, et al. Somatic and limbic cortex activation in esophageal distention: a functional magnetic resonance imaging study. Ann Neurol 1998:44:811-5.

Bushnell MC, Duncan GH, Hofbauer RK, Ha B, Chen JI, Carrier B. Pain perception: is there a role for primary somatosensory cortex. Proc Natl Acad Sci USA 1999;96:7705-9.

Casey K. Forebrain mechanisms of nociception and pain: Analysis through imaging. Proc Natl Acad Sci USA 1999;96:7668-74.

Chang C, Shyu BC. A fMRI study of brain activations during nonnoxious and noxious electrical stimulation of the sciatic nerve of rats. Brain Res 2001;897:71-81.
Cohen SR, Melzack R. Habenular stimulation produces analgesia in the formalin test. Neurosci Lett 1986;70:165-9.

D'Amour FE, Smith DL. A method for determining loss of pain sensation. J Pharmacol Exp Ther 1941;72:74-9.

Davis KD, Wood ML, Crawley AP, Mikulis DJ. fMRI of human somatosensory and cingulate cortex during painful electrical nerve stimulation. Neuroreport 1995;7:321-5.

Davis KD, Taylor SJ, Crawley AP, Wood ML, Mikulis DJ. Functional MRI of pain- and attention-related activations in the human cingulate cortex. J Neurophysiol 1997;77:3370-80.

Davis KD, Kwan CL, Crawley AP, Mikulis DJ. Functional MRI study of thalamic and cortical activations evoked by cutaneous heat, cold, tactile stimuli. J Neurophysiol 1998;80:1533-46.

Depner UB, Reinscheid RK, Takeshima H, Brune K, Zeilhofer HU. Normal sensitivity to acute pain, but increased inflammatory hyperalgesia in mice lacking the nociceptin precursor polypeptide or the nociceptin receptor. Eur J Neurosci 2003;17:2381-7.

Devinsky O, Morrell MJ, Vogt BA. Contributions of anterior cingulate cortex to behaviour. Brain 1995;118:279-306.

Dubuisson D, Dennis SG. The formalin test: a quantitative study of the analgesic effects of morphine, meperidine, brain stem stimulation in rats and cats. Pain 1977;4:161-74.

Gühring H, Gorig M, Ates M, Coste O, Zeilhofer HU, Pahl A, et al. Suppressed injury-induced rise in spinal prostaglandin E2 production and reduced early thermal hyperalgesia in iNOS-deficient mice. J Neurosci 2000;20:6714-20.

Guhring H, Tegeder I, Lotsch J, Pahl A, Werner U, Reeh PW, et al. Role of nitric oxide in zymosan induced paw inflammation and thermal hyperalgesia. Inflamm Res 2001;50:83-8.

Gyngell ML, Bock C, Schmitz B, Hoehn-Berlage M, Hossmann KA. Variation of functional MRI signal in response to frequency of somatosensory stimulation in $\alpha$-chloralose anesthetized rats. Magn Reson Med 1996;36:13-5.

Hargreaves K, Dubner R, Brown F, Flores C, Joris J. A new and sensitive method for measuring thermal nociception in cutaneous hyperalgesia. Pain 1988;32:77-88.

Harvey RJ, Depner UB, Wassle H, Ahmadi S, Heindl C, Reinold H, et al. GlyR alpha3: an essential target for spinal PGE2-mediated inflammatory pain sensitization. Science 2004;304:884-7.

Henning J, Nauerth A, Friedburg H. RARE imaging: a fast imaging method for clinical MR. Magn Reson Med 1986;3:823-33.

Hess A, Stiller D, Kaulisch T, Heil P, Scheich H. New insights into the hemodynamic blood oxygenation level-dependent response through combination of functional magnetic resonance imaging and optical recording in gerbil barrel cortex. $\mathbf{J}$ Neurosci 2000;20(9):3328-38.

Hsieh J-C, Stahle-Bäckdahl M, Hägermark Ö, Stone-Elander S, Rosenquist $\mathrm{G}$, Ingvar $\mathrm{M}$. Traumatic nociceptive pain activates the hypothalamus and the periaqueductal gray: a positron emission tomography study. Pain 1995;64:303-14.

Hudson AJ. Pain perception and response: central nervous system mechanisms. Can J Neurol Sci 2000;27:2-16.

Hyder F, Behar KL, Martin MA, Blamire AM, Shulman RG. Dynamic magnetic resonance imaging of the rat brain during forepaw stimulation. J Cereb Blood Flow Metab 1994;14:649-55.

Jones AP, Hughes DG, Brettle DS, Robinson L, Sykes JR, Aziz Q, et al. Experiences with functional magnetic resonance imaging at 1 tesla. Br J Radiol 1998;71:160-6.

Koltzenburg M, Wall PD, McMahon SB. Does the right side know what the left is doing. Trends Neurosci 1999;22:122-7.

Lorenz J, Cross DJ, Minoshima S, Morrow TJ, Paulson PE, Casey K. A unique representation of heat allodynia in the human brain. Neuron 2002;35(2):383-93.

Maihöfner C, Schmelz M, Forster C, Neundoerfer B, Handwerker HO. Neural activation during experimental allodynia: a functional magnetic resonance imaging study. Eur J Neurosci 2004;19: 3211-8. 
Malisza KL, Docherty JC. Capsaicin as a source for painful stimulation in functional MRI. J Magn Reson Imag 2001;14: $341-7$.

Malisza KL, Gregorash L, Turner A, Foniok T, Stroman PW, Allman A-A, et al. Functional MRI involving painful stimulation of the ankle and the effect of physiotherapy joint mobilization. Magn Reson Imag 2003;21:489-96.

Mao J, Mayer DJ, Price DD. Patterns of increased brain activity indicative of pain in a rat model of peripheral mononeuropathy. J Neurosci 1993;13(6):2689-702.

Meller ST, Gebhart GF. Intraplantar zymosan as a reliable, quantifiable model of thermal and mechanical hyperalgesia in the rat. Eur J Pain 1997;1:43-52.

Melzack R. Phantom limbs and the concept of a neuromatrix. Trends Neurosci 1990;13:88-92.

Neto FL, Schadrack J, Ableitner A, Castro-Lopes JM, Bartenstein P, Zieglgansberger W, et al. Supraspinal metabolic activity changes in the rat during adjuvant monoarthritis. Neuroscience 1999;94(2):607-21.

Paulson PE, Casey KL, Morrow TJ. Long-term changes in behavior and regional cerebral blood flow associated with painful peripheral mononeuropathy in the rat. Pain 2002;95(1-2):31-40.

Paxinos G, Watson C. The rat brain in stereotaxic coordinates. 4th ed. New York: Academic Press; 1998.

Petrovic P, Ingvar M. Imaging cognitive modulation of pain processing. Pain 2002;95:1-5.

Peyron R, Laurent B, Garcia-Larrea L. Functional imaging of brain responses to pain. A review and meta-analysis (2000). Neurophysiol Clin 2000;30:263-88.

Porro CA, Cettolo V, Francescato MP, Baraldi P. Temporal and intensity coding of pain in human cortex. J Neurophysiol 1998;80:3312-20.

Porro CA, Cavazzuti M, Baraldi P, Giuliani D, Panerai AE, Corazza R. CNS pattern of metabolic activity during tonic pain: evidence for modulation by beta-endorphin. Eur J Neurosci 1999;11(3): 874-88.

Porszasz R, Beckmann N, Bruttel K, Urban L, Rudin M. Signal changes in the spinal cord of the rat after injection of formalin into the hindpaw: characterization using functional magnetic resonance imaging. Proc Natl Acad Sci USA 1997;94:5034-9.

Ramos-Cabrer P, Weber R, Wiedermann D, Hoehn M. Continuous noninvasive monitoring of transcutaneous blood gases for a stable and persistent BOLD contrast in fMRI studies in the rat. NMR Biomed 2005;18(7):440-6.

Scanley BE, Kennan RP, Cannan S, Skudlarski P, Innis RB, Gore JC. Functional magnetic resonance imaging of median nerve stimulation in rats at 2.0 T. Magn Reson Med 1997;37:969-72.

Shyu BC, Lin CY, Sun JJ, Chen SL, Chang C. BOLD response to direct thalamic stimulation reveals a functional connection between the medial thalamus and the anterior cingulate cortex in the rat. Magn Reson Med 2004;52:47-55.

Spataro LE, Sloane EM, Milligan ED, Wieseler-Frank J, Schoeniger D, Jekich BM, et al. Spinal gap junctions: potential involvement in pain facilitation. J Pain 2004;5(7):392-405.

Sweitzer SM, Colburn RW, Rutkowski M, DeLeo JA. Acute peripheral inflammation induces moderate glial activation and spinal IL-1beta expression that correlates with pain behavior in the rat. Brain Res 1999;829:209-21.

Tomaz C, Graeff FG. Special issue: emotion and memory. Behav Brain Res 1993;58:1-216.

Treede RD, Kenshalo DR, Gracely RH, Jones AK. The cortical representation of pain. Pain 1999;79:105-11.

Tuor UI, Malisza K, Foniok T, Papadimitropoulos R, Jarmasz M, Somorjai R, et al. Functional magnetic resonance imaging in rats subjected to intense electrical and noxious chemical stimulation of the forepaw. Pain 2000;87:315-24.

Tuor UI, McKenzie E, Tomanek B. Functional magnetic resonance imaging of tonic pain and vasopressor effects in rats. Magn Reson Imag 2002;20:707-12.

Twining CM, Sloane EM, Milligan ED, Chacur M, Martin D, Poole S, et al. Peri-sciatic proinflammatory cytokines, reactive oxygen species, and complement induce mirror-image neuropathic pain in rats. Pain 2004;110(1-2):299-309.

Ueki M, Linn F, Hossmann KA. Functional activation of cerebral blood flow and metabolism before and after global ischemia of rat brain. J Cereb Blood Flow Metab 1988;8:486-94.

Vogt BA, Derbyshire S, Jones AK. Pain processing in four regions of human cingulate cortex localized with co-registered PET and MR imaging. Eur J Neurosci 1996;8:1461-73.

Woolfe GJ, MacDonald AD. The evaluation of the analgesic action of pethidine hydrochloride (Demerol). J Pharmacol Exp Ther 1944;80:300-7.

Zimmermann M. Ethical guidelines for investigations of experimental pain in conscious animals. Pain 1983;16:109-10. 